\title{
Kernos
}

Revue internationale et pluridisciplinaire de religion grecque antique

9 | 1996

Varia

\section{Au service des dieux et des hommes : être prêtre dans la cité de Platon}

\author{
Aikaterini Lefka
}

\section{OpenEdition \\ Journals}

Édition électronique

URL : http://journals.openedition.org/kernos/1163

DOI : 10.4000/kernos.1163

ISSN : 2034-7871

Éditeur

Centre international d'étude de la religion grecque antique

Édition imprimée

Date de publication : 1 janvier 1996

ISSN : 0776-3824

Référence électronique

Aikaterini Lefka, «Au service des dieux et des hommes : être prêtre dans la cité de Platon », Kernos [En ligne], 9 | 1996, mis en ligne le 21 avril 2011, consulté le 03 mai 2019. URL : http:// journals.openedition.org/kernos/1163; DOI : 10.4000/kernos.1163 


\section{Au service des dieux et des hommes : être prêtre dans la cité de Platon*}

Le sujet du présent travail pourrait occuper un livre entier; il s'agira donc ici de rassembler, pour une première fois, les données principales qui illustrent, ne fût-ce que de façon élémentaire, le rôle qu'un biereus est appelé à jouer dans la cité de Platon.

Cette formule, «la cité de Platon», utilisée dans le titre, fonctionne ici comme une poupée russe : elle en cache plusieurs! Dans les Lois et la République, Platon fait référence en détail à la prêtrise dans le cadre des cités idéales. Les autres dialogues, quant à eux, fournissent des informations disparates relatives soit à la fonction, soit à des personnes qui l'assument. Dans ce cas, c'est de l'« Athènes des dialogues » dont il s'agit. Enfin, il y a lieu de prendre quelquefois en considération des informations sur les fonctions des prêtres constituées par la tradition religieuse de l'époque classique, dont Platon a pu s'inspirer ou dans laquelle il introduit des innovations. C'est ainsi que s'avèrent nécessaires quelques références à des lieux historiques, comme l'Athènes où vécut Platon ${ }^{1}$.

Commençons donc le périple dans cet univers particulier des hommes et des femmes qui se consacrent «au service des dieux et des citoyens», comme le définit l'Étranger du Politique ${ }^{2}$ :

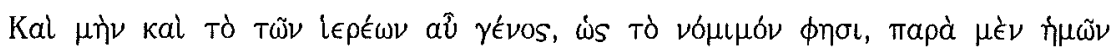

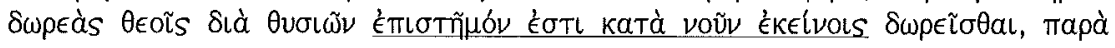

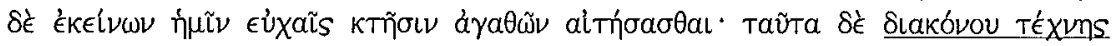

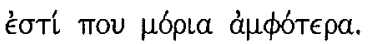

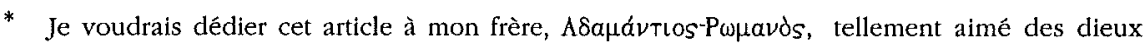
qu'il a été rappelé auprès d'eux avant son $26 \mathrm{e}$ printemps, au cours duquel j'ai fait la présente communication.

1 Sur ce dernier point surtout, je tiens à souligner la grande importance de l'étude de M. PIÉRART, Platon et la Cité grecque, Théorle et réalité dans la Constitution des "Lois ", Bruxelles, 1974 (Académie royale de Belgique, Mémotres de la Classe des Lettres, in- $8^{\circ}-2 \mathrm{e}$ série, T. LXII - Fascicule 3), où j'ai pu puiser des informations précieuses; un autre ouvrage de référence constante a été celui d'O. REVERDiN, La Religion de la Cité Platonicienne, Paris, 1945 (École Françatse d'Athènes, Travaux et Mémolres, Fasc. VI).

2 Politique, 290c 8-d 3. 
De même, la race sacerdotale sait à son tour, d'après ce qu'affirme la croyance publique, offrir aux dieux en notre nom les sacrifices qu'ils désirent et leur adresser les prières voulues pour qu'ils nous octroient leurs faveurs. Or, l'une et l'autre fonction relèvent bien, j'imagine, d'un art de service 3 .

Nous reviendrons plus tard sur cette notion du double service rendu aux dieux et aux hommes par cet intermédiaire qui connaît bien ( $\left.\dot{\epsilon} \pi \sigma T \eta \tilde{\mu} \delta_{\nu} \dot{\epsilon} \sigma \tau \iota\right)$ la façon d'accomplir les rites selon les conceptions divines (Кatà voũv ékelvoıs). Notons au préalable que le sacerdoce revêt diverses appellations et fonctions dans la religion de l'antiquité : on pouvait servir une divinité en tant que

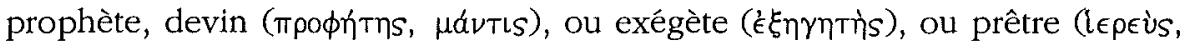

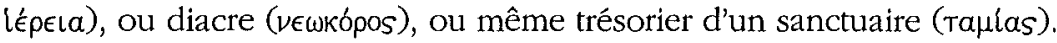

\section{Prêtres de la cité idéale et d'Athènes}

Platon accorde une place à chacune de ces fonctions dans la cité des Magnètes, projetée par les trois vieux législateurs pendant leur pélerinage de Cnossos au sanctuaire de Zeus Idaios. Cela paraît normal; leurs propos, recueillis dans les Lois, soulignent dès le départ la base religieuse sur laquelle va s'établir la future colonie : dieu, mesure de toutes choses, est le fondement même de toute bonne législation. Ses indications peuvent se manifester par des oracles, qui inspirent des législateurs vertueux (c'est déjà le cas pour les cités de Sparte et d'Athènes) ou même par des épiphanies (la Crète a joui de ce privilège, grâce à la paternité du roi Minos - rien moins que Zeus en personne). Pour la cité des Magnètes, il sera donc nécessaire de consulter le prestigieux et tout-puissant oracle de Delphes, afin de recueillir, de la bouche de Pythie, les instructions d'Apollon au sujet de l'organisation du culte de la cité (« les plus grandes, les plus belles et les premières des lois », comme les qualifie Socrate dans la République ${ }^{4}$ ).

\section{Devins-prophètes-exégètes}

Remarquable est le respect que voue Platon au rôle de la «mantique inspirée " dans la fondation de la cité; il la relie aussi plus tard à l'œuvre du législateur ${ }^{5}$. Ce qui est établi ainsi par la volonté du dieu de Delphes ou d'un autre oracle doit rester immuable ${ }^{6}$. Pourtant, aucune place n'est laissée à la mantique dite «technique », exercée par des « devins» qui, loin d'être

3 Trad. A. DIES, éd. Les Belles Lettres, 1960. Pour toutes les longues traductions de cet article, j'utilise la même édition.

4 République, IV, $427 \mathrm{~b} 2-4$.

5 Lois, VIII, 828a-b; IX, 871c-d

6 Ibid., V, 738b-d. Par ailleurs, dans la République, il est prévu qu'un éventuel changement de la législation puisse être soumis a posteriorl à l'approbation de l'oracle $(V, 461 \mathrm{e})$. 
possédés par une divinité, étaient de simples charlatans sans scrupules, des magiciens marchandant des sortilèges ${ }^{7}$. Contre eux, la législation des Magnètes prévoit des condamnations graves ${ }^{8}$, semblables à celles des athées ou des poètes qui oseraient raconter des mythes impies sur les dieux.

Quoi qu'il en soit, même les «vrais » devins ne peuvent contribuer à l'explication et à l'application des lois du culte provenant des oracles, étant eux-mêmes des instruments inconscients du savoir divin?. Platon établit donc le collège des exégètes, afin qu'il accomplisse cette œuvre difficile et importante $^{10}$. Le passage qui décrit leur désignation ${ }^{11}$ reste obscur, malgré les efforts de nombreux savants ${ }^{12}$. La procédure la plus probable semble être la suivante. Les douze tribus de la cité, divisées en trois groupes, élisent chacune un candidat. Chaque groupe retient les trois qui recueillent le plus grand nombre de voix. Les élus passent par une docimasie, identique à celle des prêtres. Elle consiste en un contrôle qui assure s'ils sont effectivement dignes d'assumer une telle fonction, à savoir : avoir plus de soixante ans, être intègre physiquement, être enfant légitime d'une famille de bonnes mours et pur de tout crime ${ }^{13}$. Les trois trios retenus sont envoyés à Delphes et c'est l'oracle qui désigne finalement un de chaque groupe. Ceux-là seront exégètes à vie. En cas de décès, le quattuor de tribus concerné doit choisir un remplaçant.

Les fonctions des exégètes consistent à intervenir, de concert avec les prêtres, prêtresses, devins et nomophylaques, à l'élaboration du calendrier du culte. Chaque année est divisée en 12 mois, chaque mois dédié au dieu protecteur de chacune des 12 tribus de la cité, et les fêtes religieuses doivent y être sciemment réparties, suivant un rite convenable ${ }^{14}$. Ils fixent encore le rituel de

7 Une image très méprisante de ces « devins vagabonds » est déjà donnée dans la République, II, $364 \mathrm{~b} 5-365 \mathrm{a} 3$.

8 Lots, X, 908d; XI, 933d-e; pour une analyse de la législation qui gère ces cas d'impiété, cf. E.A. WYLLER, Platons Gesetz gegen die Gottesleugner, in Hermes, 85 (1957), p. 292-314.

9 Platon fait une distinction entre les devins, qui sont des transmetteurs des signes divins, et les prophètes, qui savent interprëter dans un langage logique les oracles des devins (Timée, 71e 2-b 5); sur cette interaction entre inspiration et rationalité en vue de l'approche de la vérité, voir L. COULOUBARITSIS, L'art divinatoire et la question de la vérité, in Kernos, 3 (1990), p. 113-122.

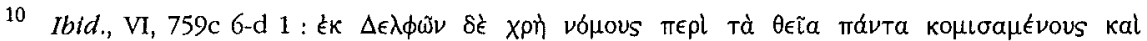

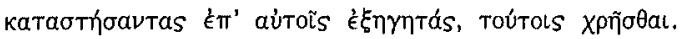

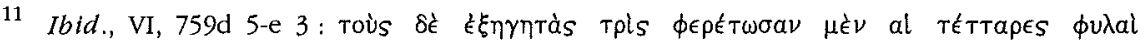

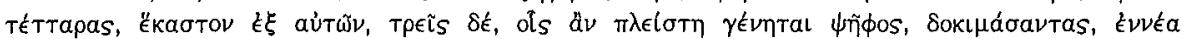

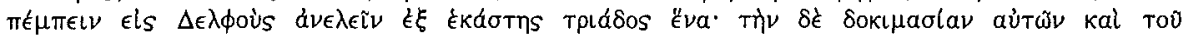

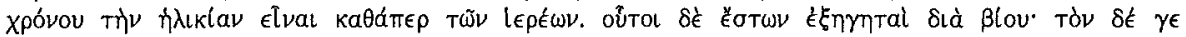

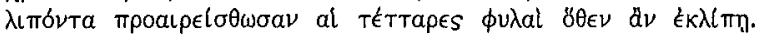

12 Cf. PIÉRART, op. cit. (n. 1), p. 327-341.

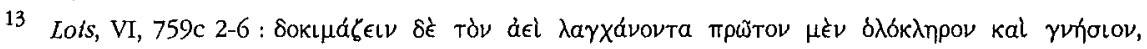

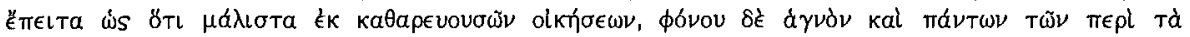

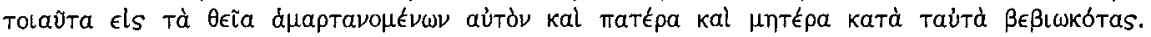

14 Ibid. VIII, 828a-d. 
purification des meurtres ${ }^{15}$ et autres crimes ${ }^{16}$, mais aussi celui des prières adéquates pour que la cité soit épargnée de tels maux ${ }^{17}$. Enfin, ils définissent les cérémonies des mariages ${ }^{18}$ et des funérailles ${ }^{19}$.

Nous savons qu'à Athènes, aux époques hellénistique et romaine, il y avait un collège de trois exégètes, désignés à vie, dont un élu à main lévée par le peuple, un pythochrestos, désigné par Delphes - tous deux choisis parmi les nobles - et un membre du genos des Eumolpides, prêtres héréditaires d'Éleusis ${ }^{20}$. Platon s'est sûrement inspiré de ce sacerdoce, mais en y précisant des limites d'âge et donnant la préférence aux qualités morales plutôt qu'à un titre de noblesse.

\section{Prêtres et prêtresses-sacristains}

Concernant la fonction des lepeĩs et des lépeıal, qui est la prêtrise proprement dite, le philosophe suit encore en grande partie les données de la tradition. Au centre de la cité des Magnètes, sur l'Acropole, est érigé le temple dédié à Hestia, Zeus et Athéna, les dieux protecteurs de l'État ${ }^{21}$. L'agora est également dotée d'un temple commun à Hélios et à Apollon ${ }^{22}$. Chacune des douze parties du pays, occupée par une tribu, possède son temple du dieu patrôos de la tribu en question; d'autres sanctuaires encore constellent la ville et la campagne ${ }^{23}$. Chacun de ces édifices sacrés doit avoir ses archontes: les prêtres qui y sont attachés. Selon la durée de leur sacerdoce et les modes de désignation, nous pouvons y distinguer trois catégories :

1. Les prêtres héréditaires, qui conservent leur titre à vie et peuvent le transmettre à leurs enfants, sous certaines conditions.

2. Ceux qui sont choisis, soit par élection, soit par tirage au sort, pour un an.

15 Ibid., IX, 865 b4-d 1.

16 Ibid., VIII, 845e 5-9.

17 Ibid., IX, 871c 3-d 2.

18 Ibid., VI, 774e 9-775a 3.

19 Ibid., IX, 873d 1-4; XII 958d 3-6.

20 Pour la famille des Eumolpides, cf. C. KERÉNYI, Eleusis, Archetypal Image of Motber and Daughter, London, 1967 (trad. R. Manheim), p. 17-18, 22-23, 48, 115.

21 Lois, V, 745b 7-8; ces dieux était des polioucboi non seulement à Athènes, mais aussi dans d'autres cités helléniques: cf. A.B. COOK, Zeus. A Study in Ancient Religion, Cambridge, 1925, III, p. 571 sq. et J. FERGUSON, Among the Gods, London, 1989, p. 173, 175.

22 Lois, VI, 778c 4-d 3 et XII, 945e 6. La disposition de ce temple, dont l'importance sera démontrée plus loin, en plein coeur de l'agora, nous suggère déjà le rôle central d'Apollon et de tout ce qu'il représente (au niveau de la politique, de l'éducation, de l'éthique) dans l'organisation de la cité. Pour ces aspects du dieu, cf. M.P. NILSSON, Les croyances religieuses de la Grèce Antique, Paris, 1955 (trad. M. Ghyka), p. 51-63.

23 Ibid., VIII, 848d 1-5; pour l'importance de la structure circulaire, divisée en douze parties et « sanctifiée », $c f$. PIÉRART, op. cit. (n. 1), p. 18-35. 
3. Les prêtres choisis pour servir le temple d'Hélios et d'Apollon, qui forment un groupe à part, dont nous reparlerons plus loin.

\section{Prêtres héréditaires}

Les patriai bierôsynai formaient une institution répandue dans l'ensemble du monde hellénique, surtout en ce qui concerne les prêtres initiateurs à des mystères $^{24}$ - comme dans le cas déjà cité de la famille des Eumolpides. Platon incite au maintien et au respect de la prêtrise héréditaire chez les Magnètes; il trouve même un compromis pour ceux qui, par naissance, auraient le droit de devenir prêtres, mais qui ne remplissent pas toutes les conditions nécessaires (de la dokimasie) : ils pourront devenir sacristains (neôkoroi) ${ }^{25}$. Ce détail n'a pas encore retenu l'attention des chercheurs. O. Reverdin et $M$. Piérart n'ont vu dans ce passage que le désir de Platon de conserver la prêtrise héréditaire, comme un exemple de plus du « souci profond que le philosophe portait à la tradition en matière religieuse ${ }^{26}$. Je pense que cette interprétation ne met pas en évidence le dilemme que le philosophe avait à affronter, ni la finesse de sa solution. Dans la cité idéale, tous les représentants d'un groupe doivent répondre aux critères fixés concernant leur qualité personnelle, indépendamment de leur origine. Platon montre à la fois son respect pour ce « sang sacralisé », en permettant à son porteur de rester dans le service des dieux, mais aussi et surtout sa fermeté en matière d'application de la justice qui gère l'ensemble de la cité : «à chacun ce qui lui sied ». Par ailleurs, M. Piérart considère qu'« il ne pourra s'agir que de sacerdoces de divinités honorées par des populations occupant le territoire où l'on fondera la colonie $»^{27}$. Or, rien n'exclut que parmi les colons aussi se trouvent des prêtres héréditaires de divinités dont le culte serait «importé » dans la nouvelle cité.

\section{Prêtres annuels}

Quant aux autres prêtres et prêtresses des Magnètes, hormis ceux d'Apollon-Hélios, ils sont désignés pour un $a^{28}$, par élection ou par tirage au sort $^{29}$. Cette dernière procédure constitue une façon de remettre à la volonté

24 Cf. Th. DE SCHEFFER, Mystères et oracles belléntques, Paris, 1943 (trad. A. Jundt), p. 63-66.

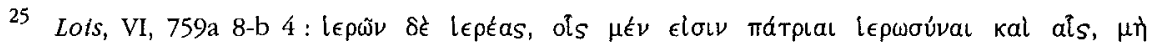

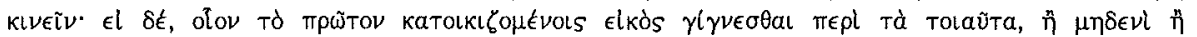

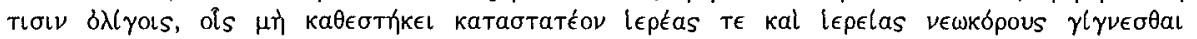
Toĩs $\theta \in o i s$.

26 PIÉRART, op. cit. (n. 1), p. 314-315 (référence également à REVERDin, op. cit. [n. 1], p. 94-95 à la p. 315, n. 3).

27 PIÉRART, ibid.

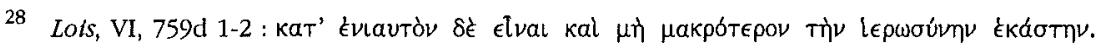

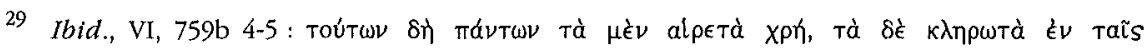

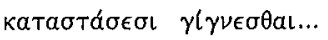


du dieu le choix de ceux qui vont le servir ${ }^{30}$. Le tirage au sort ainsi que le renouvellement annuel étaient d'ailleurs des pratiques habituelles à Athènes pour la définition des sacerdoces ${ }^{31}$. Évidemment, la docimasie valable pour les exégètes doit avoir lieu après cette désignation des candidats prêtres et prêtresses $^{32}$. La limite inférieure d'âge est égale aussi à celle des exégètes, à savoir soixante ans ${ }^{33}$. Le législateur souligne son intention de maintenir en bonne entente les citoyens-colons (dèmos) et les indigènes (mè dèmos) ${ }^{34}$ en accordant à tous le même droit de devenir prêtre pour un an ${ }^{35}$. Ces conditions d'âge attestent non seulement la gravité accordée à la fonction sacerdotale ${ }^{36}$, mais aussi la volonté du législateur d'inciter les habitants de la cité, notamment ceux qui ne pourraient pas jouir des droits civiques, à mener une vie conforme aux lois et à l'éthique établie, attitude qui sera honorée publiquement par un éventuel sacerdoce. En légiférant ainsi sur la fonction de la prêtrise, Platon réussit à en faire d'une part un facteur d'unité sociale, d'autre part un moyen d'éducation pour le maintien de la stabilité éthique et politique, toujours si précieuse à ses yeux.

Les activités exigées par un prêtre ou une prêtresse, c'est l'oracle de Delphes qui les définit à travers l'interprétation des exégètes; cependant, certaines indications, inspirées par les coutumes religieuses de toute cité hellénique, se trouvent déjà dans le texte platonicien. Les prêtres doivent veiller à l'entretien et l'ordre de leur sanctuaire ${ }^{37}$ et y accomplir des prières et des sacrifices pour la cité et les individus, le culte privé étant prohibé dans la cité des Magnètes afin de bannir toute originalité susceptible d'ébranler les croyances établies et de maintenir l'unité des citoyens face à la religion ${ }^{38}$.

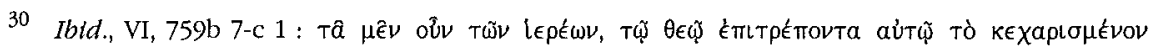

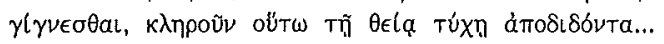

31 Cf. REVERDIN, op. cit. (n. 1), p. 61, n. 6 et 8.

32 Lots, VI, 759c 2-6 (cf. texte à la note 13).

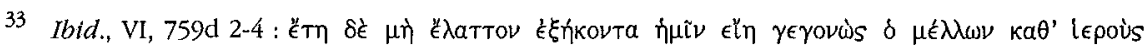

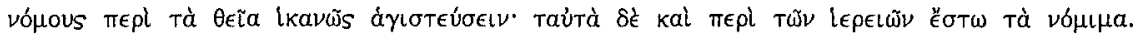

$34 \mathrm{Vu}$ les responsabilités assumées par les prêtres, il est improbable que Platon entende par mè dêmos également les métèques qui résident dans le territoire des Magnètes; leur statut est décrit dans VIII, 850a 5-d 2. Non seulement ils sont considérés comme des étrangers, mais leur séjour ne peut pas dépasser les vingt ans, sauf exception grave.

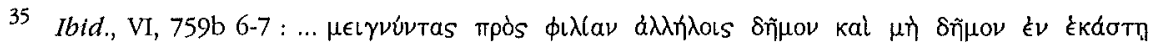

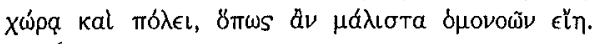

36 Platon réserve toujours les fonctions les plus importantes dans la cité - liées à la définition et l'application des règles et à la prise des décisions - aux vieillards, dont l'expérience et la sagesse garantissent la bonne gestion de l'État.

37 Lois, VI, 759a 1-8. Les prêtres sont responsables du bon fonctionnement des sanctuaires au même titre que les astynomoi et les agoranomoi, les premiers pour l'ensemble de la cité, les derniers pour l'Agora. Sur ces trois fonctions d'arcbontes repose le maintien de l'ordre du pays.

38 Lois, X, 909d 6-910 e 4. Le philosophe donne en fait une longue explication concernant la nécessité de ne pas accepter des cultes privés, en réussissant merveilleusement un mélange d'arguments théologiques, psychologiques et pragmatiques : ce n'est pas facile d'établir des 
Dans les temples sont conservés les titres de propriété des lots de terre initialement distribués aux citoyens. Leur nombre restant immuable, les prêtres et les nomophylaques sont chargés de trouver un propriétaire en cas de manque d'héritier ${ }^{39}$. Avec les neôkoroi (sacristains), au sujet desquels nous n'avons pas d'informations précises ${ }^{40}$, mais qui étaient en général des auxiliaires, les prêtres accueillent les visiteurs étrangers, dont ils seront les juges en cas de méfait ${ }^{41}$. Occasionnellement, ils participent à l'élaboration de règlements particuliers concernant leur sanctuaire ou le culte et veillent à leur application. Dans le cadre de cette dernière préoccupation, ils surveillent les hymnes et les danses pratiqués pendant les fêtes (religieuses) de la cité, notamment ceux que réalisent les jeunes ${ }^{42}$. Ces expressions artistiques doivent se conformer aux règles du culte, d'un côté parce qu'elles en font partie, de l'autre côté parce que leur rôle éducatif et spirituel est trop important pour être délaissé. Nous connaissons la place que Platon accorde à la «purification » de l'art (musique, poésie et danse), élément essentiel de la vie publique ${ }^{43}$. Significative est la mention des chants dédiés à Isis, composés une fois pour toutes et surveillés par les prêtres égyptiens; il s'agit d'un exemple à suivre, preuve que la perpétuité assurée d'un tel rite garantit la stabilité et la longévité d'une cité. En fait, ne trouvant pas parmi les fonctions habituelles des prêtres hellènes une équivalence sur ce point (alors que c'est amplement le cas pour les activités décrites précédemment ${ }^{44}$ ), Platon a recours au parallélisme avec la prêtrise

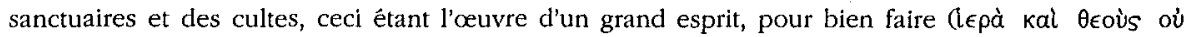

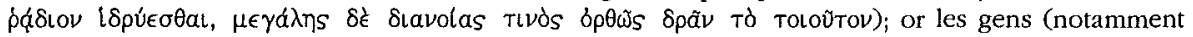
les femmes), surtout en moments de danger ou de désarroi, ont tendance à promettre un sanctuaire ou des sacrifices à la divinité qu'ils appellent en aide. Le résultat serait d'avoir des sanctuaires et des cultes divers partout, en dépit des indications déjà données par les dieux, ce qui équivaut à un délit d'impiété et entraînerait le courroux divin sur toute la cité. Le législateur peut être sûr que le

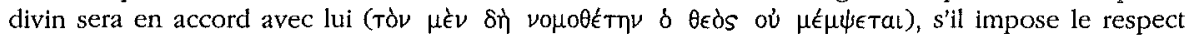
nécessaire au culte établi, en condamnant toute dérogation individuelle.

39 Ibid., V, 741 b1-d 4 et IX, 877c 8-878 b 3.

40 Platon ne fournit pas d'indications précises à leur sujet. Nous avons vu plus haut qu'ils peuvent être des personnes ayant droit à la "prêtrise héréditaire", sans pour autant pouvoir l'assumer (Lois, VI, 759 b 2-4), ce qui amène REVERDIN, op. cit. (n. 1), p. 61, à la supposition qu'ils sont les seuls à former un clergé « professionnel».

41 Lois, XII, 953a 3-b 5.

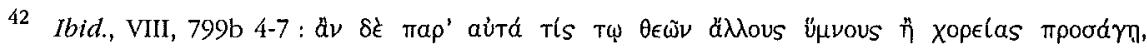

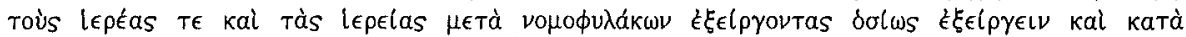

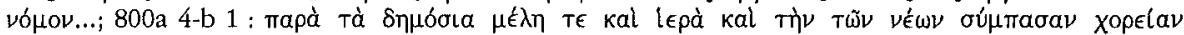

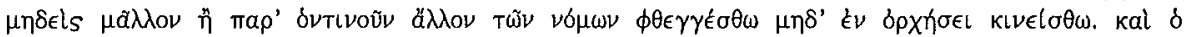

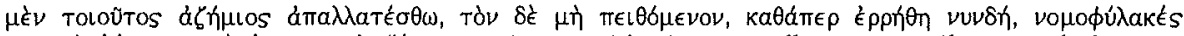

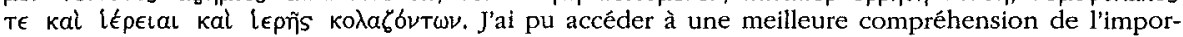
tance que Platon accorde à la fête religieuse grâce à l'article de A. MOTTE, La fête pbilosophique et le loisir des dieux, dont l'auteur a bien voulu me faire part avant sa publication; qu'il en soit vivement remercié.

43 Cf. E. MOUTSOPOUlos, La Musique dans l'ceuvre de Platon, Paris, 1959, p. 217-220, 303-319.

44

Cf. REVERDIN, op. cit. (n. 1), p. 62. 
étrangère, mais hautement estimée, de l'Égypte; et ce n'est pas la seule occasion qu'il trouvera pour le faire ${ }^{45}$.

\section{Prêtres d'Apollon et d'Hélios}

Une des magistratures les plus prestigieuses dans la cité des Magnètes est le sacerdoce très particulier des prêtres d'Apollon et d'Hélios, appelés aussi des eutbynoi (les « responsables »), car ils sont chargés de surveiller la législation, de rendre la justice, de contrôler la gestion des magistrats ${ }^{46}$.

Leur élection est originale - le texte qui la décrit est, une fois de plus, assez obscur ${ }^{47}$. Chaque année, après le solstice d'été, la cité entière se rassemble au temple d'Apollon et d'Hélios, pour dévoiler au dieu les trois meilleurs hommes de la cité; ils doivent avoir au-dessus de cinquante ans. Chacun désigne celui qu'il considère comme parfait sur tous les points ( $\pi \alpha ́ v T n$ ăpıтtov $\epsilon$ lval). Parmi les candidats proposés, on choisit par un système de vote assez compliqué les trois premiers, on les couronne de feuillage, on leur donne des prix d'excellence ( $\mathrm{dà} \mathrm{ápเ \sigma T \epsilon \tau ̃a)}{ }^{48}$ et on proclame à tous que « la cité des Magnètes, sauvée encore une fois par la divinité, présente au Soleil ses trois meilleurs citoyens et les consacre comme prémices, suivant l'usage antique, à Apollon en même temps qu'au Soleil, pour aussi longtemps qu'ils resteront fidèles au jugement porté sur eux $"{ }^{49}$. La première année de ce rituel, le peuple choisit ainsi douze euthynes, auxquels s'ajouteront trois chaque année. Le nombre total des euthynes n'est pas limité, mais ils ne peuvent exercer leurs fonctions que jusqu'à l'âge de soixante-dix ans. Il s'agit ici d'une limite d'âge révélatrice de l'importance de leurs fonctions : si Platon ne considère pas que quelqu'un puisse assumer un tel pouvoir sans avoir la cinquantaine, il se rend aussi parfaitement compte que le déclin physique d'un septuagénaire ne lui permet pas d'endosser la charge du gouvernement d'une cité.

À partir du moment de leur élection, ils sont des prêtres d'Apollon et d'Hélios, ils participent au conseil nocturne ${ }^{50}$ - institution suprême de surveillance du fonctionnement de la cité -, ils ont la présidence des théories envoyées aux fêtes et aux concours panhelléniques ${ }^{51}$. Ils sont les seuls à pou-

45 Pour un exposé plus détaillé des mentions des prêtres égyptiens, cf. A. LEFKA, Pourquoi des dieux égyptiens chez Platon?, in Kernos, 7 (1994), p. 159-168.

46 Lois, XII, 945b 3-e 3; 946d 2-e 4.

47 Ibid., XII, 945e 3-946 c 7; cf. PIÉRART, op. cit. (n. 1), p. 220-225.

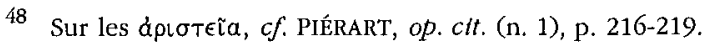

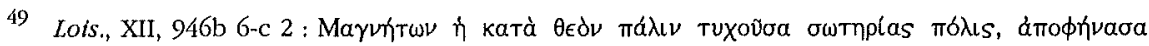

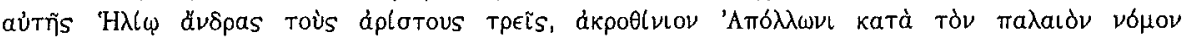

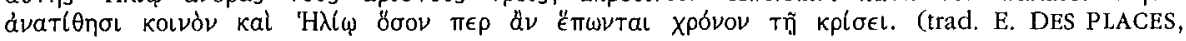
1951).

50 Ibid., XII, 951d 5-e 5.

51 Ibid., XII, 947a 1-4. 
voir porter une couronne de lauriers en public ${ }^{52}$ et demeurent tout au long de leur sacerdoce dans le temple d'Apollon ${ }^{53}$. Leurs funérailles, publiques, pleines de dignité et de splendeur, sont décrites de façon impressionnante ${ }^{54}$; le prêtre défunt est qualifié de $\mu$ akáplos (bienheureux), tout comme les héros divinisés ${ }^{55}$.

Le premier des élus de chaque année porte le titre de arcbiereôs

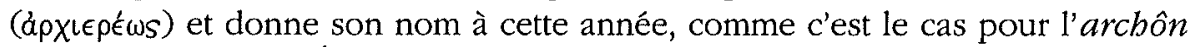
éponyme d'Athènes ${ }^{56}$. La liaison entre la prêtrise et les fonctions politiques est attestée assez fréquemment dans le monde antique. Dans le Politique, nous trouvons une référence à cette habitude, non seulement en tant qu'attribution d'une magistrature à un prêtre, mais aussi en tant que «consécration " d'un gouverneur; le roi-prêtre de l'Égypte en fournit un bel exemple, ainsi que l'archonte-roi d'Athènes ${ }^{57}$ :

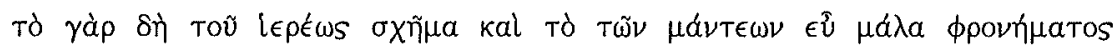

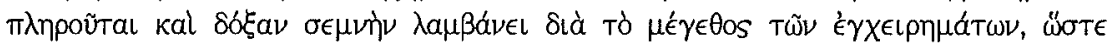

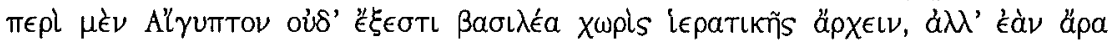

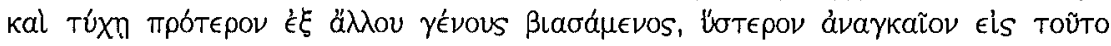

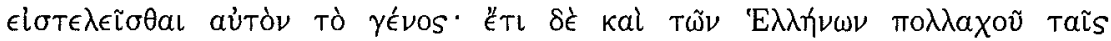

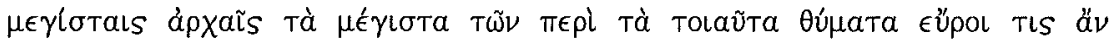
пробтатто́

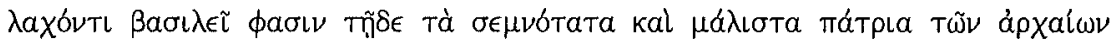

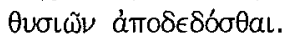

Car prêtres et devins ont des airs assurément pleins d'importance et jouissent d'un prestige en rapport avec la grandeur de leurs entreprises, tellement qu'en Égypte un roi ne peut régner s'il n'a la dignité sacerdotale, et, s'il s'est par hasard élevé par la force en partant de quelque classe inférieure, il devra finalement en venir à se faire admettre dans cette dernière caste. Chez les Grecs aussi, c'est bien souvent aux plus hautes magistratures qu'on trouverait confiée la charge d'accomplir les plus importants de ces sacrifices. Chez vous-mêmes, d'ailleurs, se vérifie non moins clairement ce que je dis, car c'est à celui des magistrats que le sort a fait roi qu'incombent ici, paraît-il, ceux de ces antiques sacrifices qui sont les plus solennels et les mieux consacrés par la tradition 58 .

\footnotetext{
52 Ibid., XII, 947a 4-5.

53 Ibid., XII, 946c 7-d 2.

54 Ibid., XII, 947b 3-e 5.

55 Cf. DES PLACES, op. cit. (n. 49), p. 56, n. 1.

56 Ibid., XII, 947a 6-b3. Il s'agit d'une habitude courante dans le monde hellénique, appliquée aussi à la prêtrise d'Hélios à Rhodes, de Zeus Naios à Dodone, et al. cf. REVERDIN, op. cit. (n. 1),

57 Politique, $290 \mathrm{~d}$ 6-e 8.

58 Trad. A. DIES, op. cit.; cf. aussi n. 1.
} p. 61, n. 12. 
Platon dote ses euthynes de plusieurs éléments courants en Égypte ou en

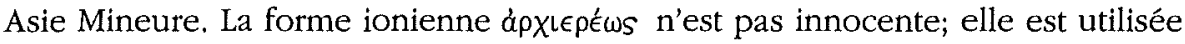
par Hérodote pour désigner le grand prêtre égyptien; on ne trouve ce titre à Athènes qu'à partir de l'époque hellénistique ${ }^{59}$. Mais le plus important est que les euthynes se distinguent nettement des autres prêtres par leur excellence morale indispensable: il s'agit non seulement de ne pas présenter d'obstacles sur le plan de l'éthique (définition «négative» d'une morale suffisante : ne pas être immoral), à l'instar des autres prêtres, mais de gagner un vrai concours de vertu (définition « positive » d'une morale extraordinaire). Ils sont les êtres les plus aptes à assurer à la cité une vie conforme à la justice et aux mesures divines, car non seulement ils sont des hommes aristoi, mais en plus ils reçoivent un savoir sur le divin «autant qu'il est possible à l'homme de connaître ${ }^{60}$.

Cet accès privilégié au sommet du savoir humain n'est pas étranger à leur consécration à Apollon et au Soleil. On ne peut s'empêcher d'établir un parallélisme entre ces dieux et l'image symbolique de l'Idée du Bien (le soleil du mythe de la caverne ${ }^{61}$ ). Par ailleurs, la nature et l'activité philosophiques, c'està-dire la prédisposition vers la recherche du bien et sa réalisation, sont considérées comme nécessaires pour les gouverneurs de la cité, autant dans les Lois que dans la République ${ }^{62}$.

Notons encore que, dans ce dernier dialogue, Platon présente déjà la poursuite philosophique de la justice et de la vertu (qu'il nomme autrement $\mathrm{els}$

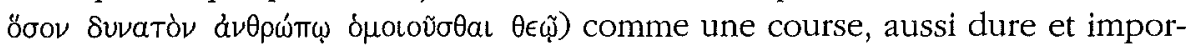
tante que celles des jeux olympiques. Le vaillant «athlète » sera couronné

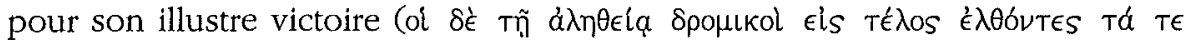
$\hat{a} \theta \lambda a \quad \lambda a \mu \beta a ́ \nu o v \sigma \iota \nu$ kal $\sigma \tau \epsilon \phi a \nu o v ̃ \nu \tau a l):$ pendant cette vie, les hommes et les dieux lui rendront des honneurs que son âme bienheureuse pourra emporter

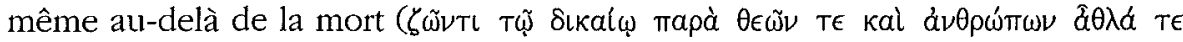

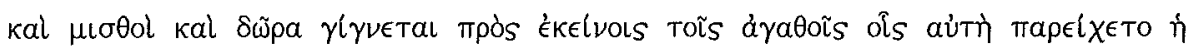

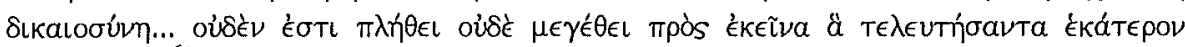
$\pi \epsilon \rho\left(\mu \epsilon \epsilon^{\prime} \iota^{\circ}\right)^{63}$. Nous pouvons aisément reconnaître la concrétisation de cette conception dans le portrait des euthynes des Lois. Il ne manque même pas l'aspect métaphysique: si le mythe d'Er clôture la République comme un

59 Cf. REVERDIN, op. cit. (n. 1), p. 61 (n. 11)-62.

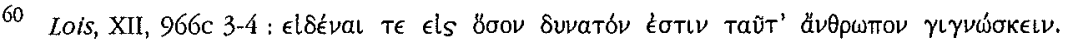

61 République, VII, 514a 2-517C 5. A. Diès et L. Gernet, dans l'introduction de la traduction des Lois par E. Des Places (Les Belles Lettres, 1951), définissent le conseil nocturne dans le même esprit : «... la science exigée de ses membres nous ramène non seulement aux preuves essentielles de l'existence et du pouvoir des dieux, mais semble rouvrir les sentiers montants que devraient gravir, au temps déjà lointain de la République, les futurs chefs de la cité, appelés à une vue synoptique des choses dans la lumière des réalités intelligibles. " (p. LXXXV).

62 Cf. R. KeNT-SPRAGUE, Plato's Pbilosopber-King, Columbia, 1976, p. 57-99.

63 République, IX, 583b 1-6; X, 613a 4-614 a 6. 
fondement « convaincant » et encourageant de cette vision ${ }^{64}$, les Lois offrent la description - curieusement? - longue et détaillée des funérailles prestigieuses des prêtres qui avaient vécu «couronnés » et partent «bienheureux».

Par conséquent, nous pouvons supposer que c'est justement ces prêtres savants qui incarnent de la façon la plus élevée possible l'idée que Platon se fait de la prêtrise, du statut d'un être à travers lequel se réalise la communication entre les dieux et les hommes.

Tout en respectant l'ensemble des personnes qui ont pu, d'une façon ou d'une autre, exercer quelque fonction sacrée, Platon manifeste un respect particulier pour ceux qui sont «possédés» par une divinité, élus comme des instruments privilégiés pour transmettre ses messages, tout en étant «actifs », conscients des moyens d'application de ce don pour le bien commun. N'oublions pas que le contact avec le divin ne peut s'effectuer que par le dépassement de ses propres limites humaines. Cette «sortie du soi » (ekstasis) est définie dans le Pbèdre comme theia mania (folie sacrée), dont on distingue quatre sortes ${ }^{65}$.

La télestique (telestikê), venant de Dionysos, est l'art de réaliser des teletai, des cérémonies rituelles. Or, toutes les cérémonies n'ont pas la même importance : dans les Lois $^{66}$, nous apprenons que plusieurs personnes écoutent, de la bouche de ceux qui ont étudié le sujet dans les teletai, des paroles qui les convainquent de la justice rendue après la mort. On se doute que la mania et le savoir téléstiques caractérisent les initiateurs à des Mystères ${ }^{67}$ et que les prêtres suprêmes qui ont «appris tout ce qu'on peut savoir sur le divin » pourraient exercer cette fonction ${ }^{68}$. La mania mantique est inspirée par Apollon, le dieu auquel les euthynes sont consacrés. La mania poétique, cadeau des Muses, est celle que la pureté des euthynes est appelée à sauvegarder par le savoir. Quant à la mania d'Éros, que Platon considère comme l'élément déclencheur de la recherche philosophique, de la quête

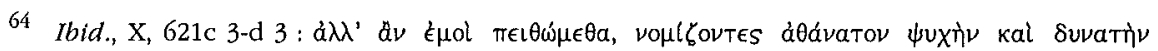

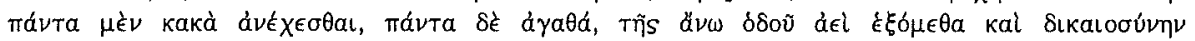

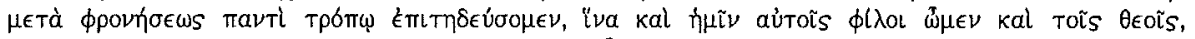

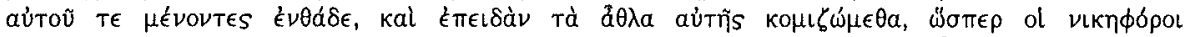

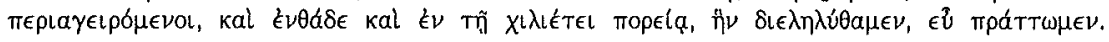

65 pbèdre, 244a 5-245c 2 et $265 \mathrm{~b} 2-5$.

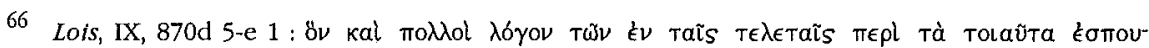

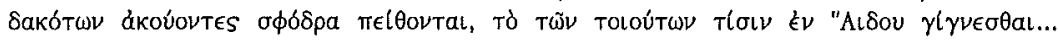

67 Le mot $T \in \lambda \in \sigma T \iota k\rangle$ ' est attesté pour la première fois dans l'œuvre de platon (deux fois seulement, dans les passages du Pbèdre cités plus haut) et sa signification a connu plusieurs versions, dont la plupart acceptent une liaison avec l'initiation aux Mystères et les rites de purification; cette constatation et un exposé philologique détaillé sur la télestique se trouvent dans l'article d'O. BALLÉRIAUX, Mantique et télestique dans le Pbèdre de Platon, in Kernos, 3 (1990), p. 35-43.

68 Cf. aussi Ménon, 81a 5-b 2, où Socrate présente la doctrine de l'immortalité de l'âme comme reçue de prêtres et de prêtresses sages, détenteurs d'un logos relatif à leur fonction. 
inassouvie du Beau, du Bien, des ailes de l'âme, peut-on imaginer que ces hommes savants et vertueux n'y auraient pas goûté ?

Nous pouvons donc retrouver la trace des liaisons apparentes ou sousjacentes que les euthynes ont avec toutes les formes du contact " passif » avec le divin. Néanmoins, ils ne restent pas que des instruments inconscients de la volonté divine, respectables certes, mais sans la valeur de l'effort personnel, comme le sont, par exemple, les devins ou les poètes ${ }^{69}$; à l'instar des philosophes, la recherche du savoir et son application couronnent ces élus et justifient la place exceptionnelle que Platon leur accorde.

\section{Prêtres dans les dialogues}

D'autre part, certains personnes des dialogues sont dotées d'une fonction qui relève du sacré, qu'il s'agisse effectivement de prêtre et de poète missions traditionnellement liées à des divinités -, ou de quelque personnage auquel Platon attribue métaphoriquement un caractère divin. Il serait intéressant de voir de près la façon dont ces personnes sont présentées, afin de mieux comprendre l'attitude de Platon face au sacerdoce.

Euthyphron, dans le dialogue homonyme, entreprend d'« expliquer » à Socrate ce qu'est le pieux (bosion); il se considère comme une autorité en la matière ${ }^{70}$, étant pourvu du don de divination. Il est vrai que, comme c'était le cas de Cassandre jadis, tout le monde se moque de lui dans l'Ecclésia une fois qu'il leur parle "des choses divines »; on le traite comme un fou (mainomenou) même s'il n'a jamais été démenti. Pourtant, il attribue ce genre d'attitude à la jalousie des gens et sympathise avec le sort de Socrate, que les Athéniens veulent amener au tribunal en tant qu' « innovateur religieux » parce qu'ils ne comprennent pas son daimonion ${ }^{71}$. Le philosophe lui rend la politesse en acceptant clairement qu'il a à faire à un devin ${ }^{72}$. Or, il ne s'agit que d'ironie, tout comme l'admiration manifestée devant le savoir prétendu d'Euthyphron; attitude qui persiste jusqu'à la fin du dialogue, puisque la discussion a démontré l'ignorance et les contradictions de notre devin ${ }^{73}$. Cette attitude peu respectueuse de Socrate et la personnalité caricaturale d'Euthyphron peuvent, à première vue, nous étonner. Cependant, l'attitude des citoyens face à ce personnage, l'information qu'Euthyphron a des rapports avec Naxos ${ }^{74}$ (un des centres importants du culte dionysiaque, situé près de Paros, où le Pythagorisme floris-

${ }^{69}$ Les hommes possédés par la divinité $(\epsilon u \theta \epsilon o \mathrm{o})$, que ce soit des poètes ou des devins, ressemblent à des pierres attirées par un aimant; une fols magnétisées, elles attirent les autres. C'est ainsi que le divin opère à travers eux et malgré eux. Cf. Ion, 533d 1-535a 2.

70 Eutbypbron, 4 e $4-5$ a 2.

71 Ibid., 3b 5-c 5.

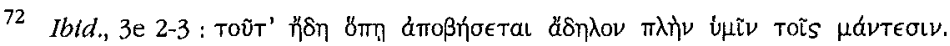

73 Ibid., 15c 12-16a 4 .

74 Ibid, 4c 4. 
sait) et, par-dessus tout, les principes manifestement contraires à la morale courante qui le poussent à traduire au tribunal son propre père, nous obligent à le considérer comme un représentant de croyances «marginales ${ }^{75}$. Nous avons plutôt ici un exemple concret des « devins charlatans", condamnables, comme on l'a vu, dans la République et dans les Lois.

Par contre, quand il s'agit d'une personne vraiment inspirée par la divinité, même si son savoir est prouvé également en défaut, le respect qui lui est dû reste intact; c'est le cas d'Ion, aède éminent, face auquel Socrate adopte une attitude enjouée, mais sans dureté, pour le convaincre qu'il est divin (theios) plutôt que savant ${ }^{76}$.

Pourtant ces deux attributs ne sont pas toujours séparés. Pour ne citer qu'un exemple, l'Étranger éléate du Sopbiste est qualifié par Théodore d'« homme divin(theios); car il appelle ainsi tous les philosophes $»^{77}$.

Dans le Banquet, c'est d'une autre «étrangère » dont il est question, qui

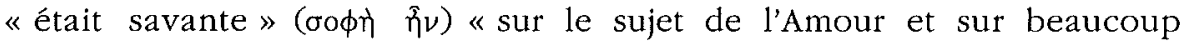
d'autres » : Diotime, prêtresse de Mantinée, venue à Athènes pour effectuer un sacrifice qui éloigne une peste de la cité pendant dix ans ${ }^{78}$, devient le maître de Socrate (le seul qu'il reconnaît comme tel) pour «l'instruire sur les choses de l'Amour ${ }^{79}$. Socrate raconte tout ce que Diotime lui avait enseigné sur la nature du daimon Éros, sur les émotions qu'il crée chez les humains et sur leurs résultats ${ }^{80}$. Cette première partie, ta érôtika, est assez simple :

Ce sont là, je le reconnais, celles des choses d'amour au mystère desquelles, même toi, Socrate, tu peux probablement être initié(myètheiès). Quant à l'initiation parfaite et à la révélation (ta de telea kai epoptika), qui aussi bien sont le but final de ces premières instructions à condition qu'on suive la bonne voie, je ne sais pas si elles seraient à ta portée. Bien sûr, je parlerai, dit-elle, et même je m'y donnerai sans la moindre réserve ! À toi d'essayer de me suivre dans la mesure de tes moyens ${ }^{81}$.

75 Cf. J. BARNES, Plato's Eutbypbro, Apolgy of Socrates and Crito, Oxford, 1954', p.5-34. Par contre, M. CROISET, dans la notice de sa traduction d'Euthyphron, Les Belles Lettres, $1970^{10}$, p. 178180 , considère qu'il s'agit d'un « docteur en théologie traditionnelle", choisi par Platon «pour mieux montrer les défauts d'une religion étroitement attachée à cette théologie ». Mais s'il est vrai que Platon trouve ici une belle occasion de démontrer le ridicule et même les dangers d'une croyance à la morale des dieux, telle qu'elle se présente dans les mythes des poètes, il est improbable que la société de l'antiquité adoptait une telle attitude dans sa majorité. Se tourner contre son propre père, par exemple, serait criminel en tout cas.

76 Ion, 541e 1-542 b 4.

77 Sophiste, $216 \mathrm{~b} 9-\mathrm{c} 1$.

78 Il s'agit de la peste qui a finalement éclaté en 430 av. J.-C.

79 Banquet, 201d 1-5.

80 Ibid., 201d 5-209e 4 .

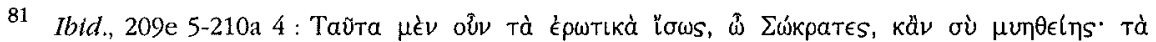

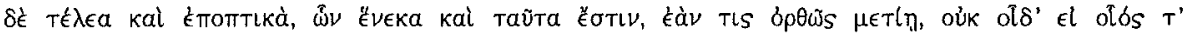


La fin du discours de Diotime explique comment l'Éros peut amener le philosophe à la contemplation du Beau et du Bien, au bonheur parfait. Socrate déclare qu'il en est convaincu (pepeismai) et qu'il essaie désormais à son tour de convaincre les autres aussi. ${ }^{82}$ Nous retrouvons ici le même effet qu'ont les lois divines sur les citoyens : ils ne s'agit pas d'imposition, mais de libre acceptation de la véritée ${ }^{83}$. La peithô était encore présente plus haut, chez les initiateurs passés par des téletai; et la télestique semble familière à Diotime, qui non seulement se présente comme initiatrice de Socrate $^{84}$ mais aussi comme purificatrice de la cité. L'existence historique de la prêtresse est remise en question par L. Robin ${ }^{85}$; par contre, R. Godel ne trouve pas du tout improbable la vérité historique des paroles de Socrate. Il soutient même que Diotime ne pouvait être qu'une hiérophante d'Apollon Pythien, le dieu purificateur par excellence $^{86}$. Quoi qu'il en soit, telle qu'elle apparaît dans le dialogue, elle semble s'approcher de l'idée du sacerdoce qu'incarnent les euthynes, autant par sa sagesse, par sa fonction d'initiatrice, liée à la mania philosophique d'Éros et à la télestique, que par les interventions salvatrices qu'elle rend à toute une cité (directement, en rétablissant le rapport harmonieux avec les dieux, et indirectement, via l'éducation de Socrate, qui, à son tour, répand les connaissances reçues parmi les citoyens).

Quant à Socrate même, le prototype du philosophe pour Platon, non seulement il entreprend cette fonction d'initiateur philosophique inspiré par Éros, mais en plus il se déclare serviteur (bomodoulos) et consacré à Apollon (bieros tou autou theou), dont il avoue avoir reçu le don de mantique - tout comme les cygnes ${ }^{87}$. Socrate s'attribue des capacités divinatoires à plusieurs reprises ${ }^{88}$; en plus, il considère toute son activité philosophique comme un accomplissement de la volonté du dieu, dévoilée à lui par des signes. Dans l'Apologie, c'est l'oracle de Delphes qui l'incite à la recherche de la sagesse ${ }^{89}$

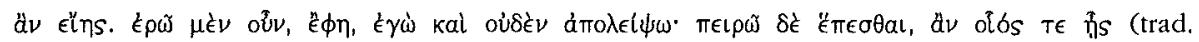
L. ROBIN, 1958)

82 Ibid., 212b 1-3.

83 Pour l'identification de la croyance $(\pi \iota \sigma \tau \epsilon \in \dot{\epsilon} \epsilon \nu)$ à la conviction ( $\pi \epsilon(\theta \epsilon \sigma \theta a \iota)$ dans l'esprit religieux de l'antiquité, $c f$. U. VON WILAMOWITZ-MOELLENDORF, Der Glaube der Hellenen, Basel, $1959^{3}$, I, p. 12-14.

84 Cf. Banquet, commentaire de L. ROBIN, op. cit. (n. 60), p. 67, n. 4; p. 68, n. 2.

85 Ibid., p. XXII-XXVII.

86 Cf. R GODEL, Socrate et Diotime, Paris, 1955, p. 31-32.

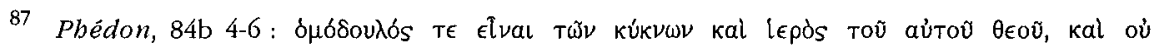

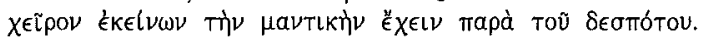

88 Phèdre, 242c 3-6; sur la relation entre le discours philosophique et la parole divinatoire de Socrate dans ce dialogue, cf. R. BODÉüs, "Je suis devin" (Phèdre, 242 c), in Kernos, 3 (1990), p. 45-52. Il y a encore le rêve prémonitoire de Socrate en prison (Criton, 43c 5-44b 4), son daimonion qui l'empêche d'avancer vers des actes qui auront des conséquences mauvaises (Apologie, 31c 7-d 6)...

89 Apologie, 20e 8-b 9. 
et, dans le Phédon, il fait état du rêve qui l'appelait souvent à « faire de la musique et à travailler ${ }^{90}$; Socrate a toujours considéré la philosophie comme la meilleure "musique" - l'éducation la plus élevée et la recherche de l'harmonie du cosmos. Enfin, il n'hésite pas à déclarer à ses juges que, pendant toute sa vie, il a obéi aux ordres d'Apollon, au moins autant qu'il a pu les interpréter, et qu'il estime que son service du dieu (tô theô bypèresian) a fait le plus grand bien à la cité ${ }^{91}$. C'est ainsi qu'il peut affronter sa mort avec le calme de celui qui a accompli son devoir envers lui-même, les hommes et les dieux, qu'il va rejoindre bientôt, comme un «bienheureux $»^{92}$.

Nous retrouvons donc réunis les thèmes du respect dû à la volonté divine, de communication (à la fois «passive» et «active») avec le divin, de l'acquisition d'un savoir difficilement accessible au grand nombre des mortels et de son application en tant qu'enseignement pour le bien de la cité, du retour bienheureux de l'âme auprès des dieux, autant chez les prêtres-magistrats que Platon met au sommet de la hiérarchie des sacerdoces et du pouvoir public que chez Socrate en personne. Il y aurait peut-être lieu de concevoir l'idée que Platon se fait de la prêtrise, sous sa forme la plus excellente, comme le troisième attribut de la personnalité modèle du philosophe-roi, de l'être humain qui, élu pour son excellence, s'approche le plus possible du divin, tout en mettant en œuvre ses qualités pour le bien de la communauté civique.

Université de Liège

Aikaterini LEFKA

Philosophie

32, place du 20-Août,

B - 4000 LIÈGE

90 Pbédon, 60e 4-61a 4.

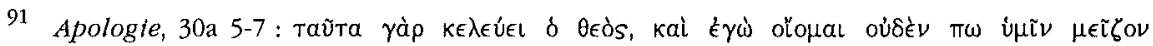

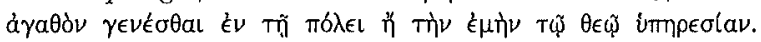

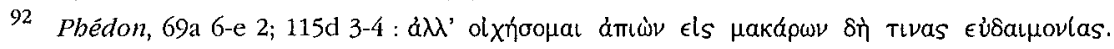

